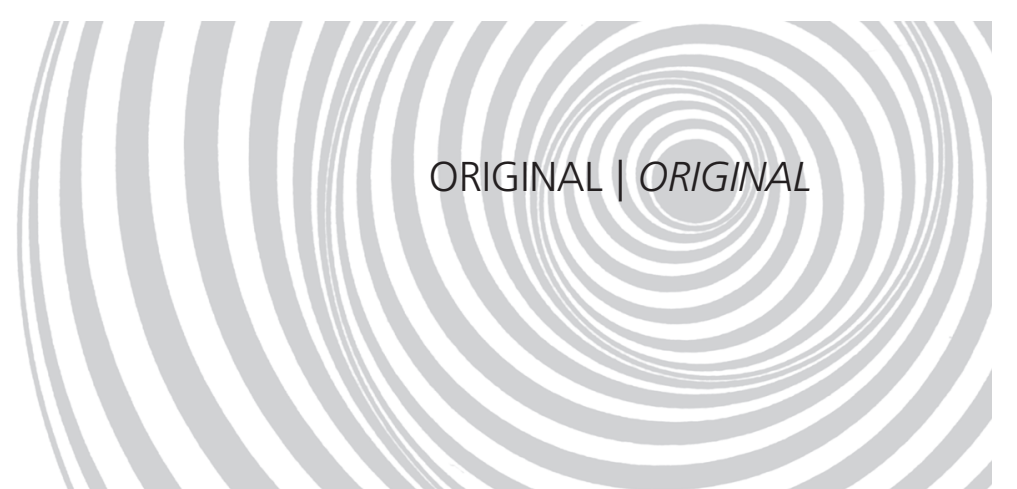

\title{
Hipertensão arterial e orientação domiciliar: o papel estratégico da saúde da família'
}

\author{
Hypertension and educational home visits: \\ the strategic role of family healthcare
}

\author{
Amanda Gomes RIBEIRO2 \\ Rosângela Minardi Mitre COTTA² \\ Luciana Saraiva da SILVA ${ }^{2}$ \\ Sônia Machado Rocha RIBEIRO² \\ Cristina Maria Ganns Chaves DIAS² \\ Sandra Minardi MITRE ${ }^{3}$ \\ Maria Cezira Fantini NOGUEIRA-MARTINS ${ }^{4}$
}

RE S U M O

\section{Objetivo}

Analisar a importância, a efetividade e as limitações de estratégias participativas de educação em saúde sobre a problemática da adesão ao tratamento da hipertensão arterial.

\section{Métodos}

Estudo de caso, intervencional, de abordagem qualiquantitativa com duração de 4 meses. Participaram do estudo 27 mulheres portadoras de hipertensão arterial, com idade entre 45 e 60 anos e cadastradas no Programa de Saúde da Família de Porto Firme, Minas Gerais. Foram comparadas duas modalidades de intervenção visando à orientação de mudanças dietéticas indicadas no tratamento da hipertensão arterial: uma baseada em atividades educativas em grupo, realizadas na Unidade de Atenção Primária à Saúde; e outra combinada pelas atividades educativas em grupo e por orientações domiciliares. Os dados foram obtidos por grupos focais e entrevistas individuais. Para avaliar os dados qualitativos relacionados à adesão, foram analisados, antes e após a intervenção, o peso corporal, o índice de massa corporal, a circunferência de cintura, a pressão arterial, a glicemia e o consumo alimentar. Os dados foram examinados por análise de conteúdo e estatística descritiva. As variáveis coletadas antes e depois da intervenção foram analisadas pelos testes não-paramétricos de Wilcoxon e Mann Whitney.

\footnotetext{
1 Artigo elaborado a partir da dissertação de AG RIBEIRO, intitulada "A problemática da adesão às ações e atividades em saúde de portadores de hipertensão arterial: (re)pensando estratégias em nutrição e saúde". Universidade Federal de Viçosa; 2012. Apoio: Fundação de Amapro à Pesquisa do Estado de Minas Gerais, processo no APQ-00197-09.

2 Universidade Federal de Viçosa, Departamento de Nutrição e Saúde. Av. P.H. Rolfs, s/n., Campus Universitário, 36570-000,

Viçosa, MG, Brasil. Correspondência para/Correspondence to: RMM COTTA. E-mail: <rmmitre@ufv.br>.

3 Faculdade de Ciências Médicas de Minas Gerais, Fundação Educacional Lucas Machado. Belo Horizonte, MG, Brasil.

4 Secretaria de Estado da Saúde de São Paulo, Instituto de Saúde. São Paulo, SP, Brasil.
} 
272 | AG RIBEIRO et al.

\section{Resultados}

As duas estratégias de educação em saúde tiveram efeito positivo sobre a adesão às orientações nutricionais, o que favoreceu mudanças nos hábitos alimentares e na percepção das mulheres em relação à problemática da doença.

\section{Conclusão}

A orientação domiciliar destacou-se como importante indutor da consciência sanitária e como fator de envolvimento dos familiares no processo terapêutico, facilitando a adesão ao tratamento da hipertensão arterial.

Termos de indexação: Educação em saúde. Hipertensão arterial. Nutrição. Saúde da família.

\section{A B S T R A C T}

\section{Objective}

This study assessed the limitations of two educational strategies and their efficacy in increasing adherence to hypertension treatment.

\section{Methods}

This interventional case study of qualitative and quantitative nature included 27 hypertensive women aged 45 to 60 years, and lasted four months. The women were enrolled in the Family Health Program of Porto Firme, Minas Gerais. Two diet education interventions to reduce blood pressure were compared: one consisted of classes given at the primary healthcare unit, and the other, of classes given at the primary health care unit plus home visits. Data were collected though focus groups and individual interviews. Body weigh, body mass index, waist circumference, blood pressure, blood glucose and food intake were determined before and after the intervention to investigate possible association with adherence. The data were then submitted to content analysis and descriptive statistics. The variables collected before and after the intervention were compared with the non-parametric Wilcoxon and Mann Whitney tests.

\section{Results}

The two educational strategies promoted adherence to appropriate food habits and improved the women's perception of hypertension-related problems.

\section{Conclusion}

Home visits proved to be important for promoting good hygiene practices and getting the family members involved in the therapeutic process, encouraging patients' adherence to hypertension treatment.

Indexing terms: Health education. Hypertension. Nutrition. Family health.

\section{N T R O D U Ç Ã O}

A Hipertensão Arterial Sistêmica (HAS) é um grave problema de saúde pública no Brasil e no mundo e um fator de risco independente para doenças cardiovasculares e renais ${ }^{1}$. Apesar do risco que a HAS representa, a adesão à terapia anti-hipertensiva ainda é insatisfatória e permanece como desafio aos serviços de saúde e às políticas públicas, em especial na Atenção Primária à Saúde (APS). A grande maioria dos portadores de HAS não tem sua pressão controlada de forma efetiva, o que pode ser explicado pela baixa adesão ao tratamento. Estima-se que, entre os pacientes em tratamento, $75 \%$ a $92 \%$ não consi- gam manter a pressão arterial em níveis satisfatórios ${ }^{2}$.

A adesão à terapêutica por parte dos portadores de doenças crônicas como a HAS tem sido discutida como um processo complexo e multifatorial. Sob o ponto de vista do indivíduo, a adesão relaciona-se ao reconhecimento, à aceitação e à adaptação à condição de saúde, bem como à identificação de fatores de risco no estilo de vida adotado e ao desenvolvimento do autocuidado e de hábitos e atitudes saudáveis³.

Como fatores dificultadores da adesão, destacam-se a falta de informação sobre a doença, a passividade do indivíduo em relação aos 
profissionais de saúde e à escolha do esquema terapêutico, e as representações negativas relacionadas à doença e ao tratamento ${ }^{3,4}$. No caso da HAS, vários motivos são apontados como causa para a resistência à mudança de hábitos de vida, dentre eles o curso assintomático da doença, a subestimação de suas reais consequências e a dificuldade de mudança de padrões comportamentais construídos ao longo do tempo ${ }^{5}$.

Logo, as metas da educação em saúde para o indivíduo portador de HAS devem incluir a apropriação de meios para o desenvolvimento de seu autocuidado e autonomia, a ampliação de seu nível de conhecimento e apreensão sobre os processos de saúde-doença-adoecimento e o desenvolvimento de estratégias para seu empoderamento e libertação ${ }^{6,7}$.

Em relação à terapêutica não medicamentosa, a alimentação e a nutrição ocupam lugar de destaque na mudança de estilo e hábitos de vida dos indivíduos com HAS; a educação nutricional deve desenvolver-se rumo a estratégias mais saudáveis de viver, cuidar e ser, auxiliando esses indivíduos na superação de mitos e crenças, e no desenvolvimento de valores, percepções e atitudes ativas à saúde ${ }^{5,8}$. A participação da família nas mudanças da rotina diária do núcleo familiar, sobretudo em relação à alimentação, tem papel de fundamental importância para viabilizar a adesão ao tratamento não farmacológico da $\mathrm{HAS}^{7,8}$,

O objetivo deste estudo foi analisar a importância, a efetividade e as limitações de estratégias de educação em saúde, relacionadas às orientações dietéticas, no enfrentamento da problemática da adesão ao tratamento da HAS, no contexto do Programa de Saúde da Família (PSF).

\section{M É T O D O S}

Trata-se de um estudo de caso, intervencional e de abordagem qualiquantitativa, denominado triangulação de métodos ${ }^{9}$. O estudo foi realizado na UAPS da área urbana de Porto Firme (MG), no período de abril a agosto de 2009.

Participaram do estudo 27 mulheres entre 45 e 60 anos, com diagnóstico confirmado de
HAS, sem acompanhamento nutricional e sem condições clínicas graves, após assinarem o termo de consentimento livre e esclarecido.

As mulheres selecionadas para o estudo foram subdivididas por sorteio em dois grupos que participaram de duas estratégias de educação em saúde e nutrição (Quadro 1). Um grupo participou de oficinas mensais de educação em saúde, e o outro grupo, além da participação nessas oficinas, recebeu orientação individualizada e familiar no domicílio por profissional nutricionista.

Os dados sobre o perfil sociodemográfico e as modificações nos hábitos alimentares foram coletados em entrevistas individuais e analisados por meio de estatística descritiva. Os dados qualitativos foram obtidos em entrevistas individuais nos domicílios e em dois grupos focais. O corpus de análise dos dados qualitativos foi examinado por meio da análise de conteúdo, descrita por Minayo $^{10}$ e Bardin ${ }^{11}$.

Para a triangulação de métodos, além da análise qualitativa, foram analisados parâmetros quantitativos, antes e após a intervenção, incluindo peso corporal, Índice de Massa Corporal (IMC), Circunferência de Cintura (CC), Pressão Arterial (PA), níveis de glicemia, colesterol total e frações e análise do consumo alimentar por meio de um Questionário de Frequência de Consumo Alimen$\operatorname{tar}(\mathrm{QFCA})^{12}$.

As variáveis antropométricas foram coletadas durante as oficinas. O peso corporal foi obtido por meio de balança eletrônica, com capacidade de $180 \mathrm{~kg}$; e a estatura foi aferida por meio de antropômetro portátil de acordo com as técnicas propostas por Jellife ${ }^{13}$. O IMC foi calculado e classificado segundo critérios da Organização Mundial da Saúde (OMS) ${ }^{14}$. A CC foi aferida por meio de fita inextensível na menor circunferência observada e classificada de acordo com os critérios da OMS ${ }^{15}$. A PA foi aferida e classificada de acordo com os procedimentos e parâmetros recomendados pelas $\vee$ Diretrizes Brasileiras de Hipertensão Arterial ${ }^{16}$. As variáveis bioquímicas foram analisadas por meio de técnicas de rotina clínica, com as participantes orientadas previamente sobre $o$ jejum de 12 horas. 
Quadro 1. Conteúdo das oficinas de educação em saúde e visitas domiciliares realizadas com mulheres portadoras de hipertensão arterial do município de Porto Firme (MG), 2009.

\begin{tabular}{|c|c|c|}
\hline $\begin{array}{l}\text { Oficina 1: Hipertensão Arterial: con- } \\
\text { ceito, fatores de risco e medidas dieté- } \\
\text { ticas do tratamento }\end{array}$ & $\begin{array}{l}\text { Vídeo Ministério da Saúde: Hiper- } \\
\text { tensão Arterial. 15'. Palestra: } 30^{\prime}\end{array}$ & $\begin{array}{l}\text { Visita 1: Identificar possíveis exageros no uso de óleo, sal } \\
\text { e açúcar e auxiliar na mudança de hábitos relacionados } \\
\text { ao uso desses gêneros }\end{array}$ \\
\hline $\begin{array}{l}\text { Oficina 2: Consumo de gordura/óleo, } \\
\text { açúcar e sal de adição: recomenda- } \\
\text { ção e perigos do consumo excessivo }\end{array}$ & $\begin{array}{l}\text { Palestra: } 30^{\prime} \text {. Dinâmica: demons- } \\
\text { tração prática de uso correto de } \\
\text { óleo, açúcar e sal }\end{array}$ & $\begin{array}{l}\text { Visita 2: Identificar os principais gêneros alimentícios } \\
\text { adquiridos no domicílio e realizar as orientações neces- } \\
\text { sárias para melhora dos hábitos alimentares, de acordo } \\
\text { com a dieta habitual da família }\end{array}$ \\
\hline $\begin{array}{l}\text { Oficina 3: Consumo de frutas, verdu- } \\
\text { ras e legumes (FV\&L): importância e } \\
\text { recomendações }\end{array}$ & $\begin{array}{l}\text { Palestra: } 30^{\prime} \text {. Dinâmica: demons- } \\
\text { tração prática - porção diária re- } \\
\text { comendada de FV\&L. 15' }\end{array}$ & $\begin{array}{l}\text { Visitas } 3 \text { e 4: Reforçar as orientações dadas nas visitas an- } \\
\text { teriores, esclarecer as dúvidas e realizar os ajustes neces- } \\
\text { sários de acordo com as necessidades específicas de cada } \\
\text { família }\end{array}$ \\
\hline $\begin{array}{l}\text { Oficina 4: Pirâmide Alimentar Brasi- } \\
\text { leira - conceitos fundamentais: pro- } \\
\text { porcionalidade, moderação e varieda- } \\
\text { de }\end{array}$ & $\begin{array}{l}\text { Palestra: } 30^{\prime} \text {. Dinâmica: reconhe- } \\
\text { cendo os grupos alimentares. 15' } \\
\text { Palestra: } 40^{\prime}\end{array}$ & \\
\hline $\begin{array}{l}\text { Oficina 5: Atividade física: benefícios } \\
\text { e importância para redução/manuten- } \\
\text { ção do peso }\end{array}$ & & $\begin{array}{l}\text { Visita 5: Discussão e reflexão sobre as principais mudan- } \\
\text { ças ocorridas na alimentação da participante e também } \\
\text { na da família, reforçando as conquistas alcançadas e esta- } \\
\text { belecendo as metas a serem superadas }\end{array}$ \\
\hline
\end{tabular}

Os dados de consumo alimentar obtidos pelo QFCA foram analisados quantitativamente por meio de escores de consumo individual, de acordo com o método proposto por Fornés et al. ${ }^{17}$. Para a análise dos dados, foram utilizados os softwares Excel for Windows 2007 e Statistical Package for the Social Sciences for Windows (Version 18.0; SPSS Inc, Chicago, III). As variáveis quantitativas foram analisadas pelos testes não-paramétricos de Wilcoxon e Mann Whitney, considerando nível de significância estatística de $p<0,05$.

O trabalho foi aprovado pelo Comitê de Ética em Pesquisa da Universidade Federal de Viçosa (UFV), protocolo $n^{\circ}$ 030/2009, em consonância com a Resolução 196/96 do Conselho Nacional de Saúde.

\section{RESULTADOSE DISCUSSÃO}

A média de idade das participantes foi de 53 anos. A maioria (85\%) tinha ensino fundamental incompleto. Quanto ao estado civil, 96\% eram casadas ou tinham relacionamento estável, e $4 \%$ eram divorciadas. Apenas $11 \%$ tinham trabalho formal com vínculo empregatício, enquanto $43 \%$ eram "do lar", $21 \%$, aposentadas ou pensionistas, e $35 \%$ tinham trabalho informal ou eram trabalhadoras rurais sem vínculo empregatício. A renda familiar de cerca de $80 \%$ das mulheres se concentrava entre um e três salários-mínimos; 53\% delas contribuíam com a renda familiar.

O material obtido por meio das entrevistas e dos grupos focais foi organizado em torno de dois grandes temas:

1. A percepção das participantes sobre as atividades educativas: do individual ao coletivo a formação do vínculo.

O processo ensino-aprendizagem, como ação que gera "empoderamento", é um aspecto marcante na fala das participantes, que salientaram a importância das estratégias de educação em saúde e nutrição no aumento da percepção de si mesmas, na conscientização e no controle do próprio corpo e na apreensão das questões relativas ao cuidado dietético na HAS ${ }^{18}$. 
Eu não tinha muita consciência, né, do efeito dos alimentos no organismo. A partir do momento que você (a pesquisadora) fez as palestras e as visitas, eu aprendi muito (1).

Nas atividades educativas realizadas, ficou evidente que as mulheres foram transformando-se em reais sujeitos da construção e reconstrução do "saber", apreendido em sua "razão de ser" e como um processo libertador. O acesso às informações pôde emancipá-las de uma postura passiva e de dependência, favorecendo a autonomia e capacitando-as a vislumbrar novas atitudes, facilitadoras de mudanças nos hábitos alimentares ${ }^{6,18}$.

Antes a gente num mudava porque num tinha ninguém pra conversar com a gente, falar pra gente o que era bom ... então a gente não sabia de nada, é a mesma coisa que você ser um ignorante, ser cego, andar cego no escuro. E no explicar a gente passa a saber de tudo (4).

As mulheres que receberam as visitas domiciliares relataram que:

Em casa é melhor explicado ainda. Em grupo... mostra lá pra gente slide, cartaz ou oral... a pessoa num guarda tudo aquilo ali... a prática junto com a gente, individual ali em casa, é muito melhor (1).

De fato, as mulheres que receberam a visita domiciliar atribuíram maior importância à atenção individualizada e no domicílio. Apesar de reconhecerem o valor das atividades em grupo, destacaram as vantagens das visitas e das orientações no próprio lar, em detrimento das limitações inerentes às atividades em grupos maiores. Acredita-se que isso tenha ocorrido porque no grupo que recebeu a orientação individualizada e no locus onde as pessoas vivem e preparam suas refeições, direcionou-se o processo de ensino-aprendizagem de acordo com as dificuldades específicas de cada participante e grupo familiar. A entrada no lar dessas mulheres propiciou o estreitamento do laço e o fortalecimento do vínculo na relação entre o pesquisador e os sujeitos participantes e sua rede social. Na problemática da adesão, o vínculo constitui um princípio importante no cuidado, pois estabelece uma relação de confiança, diálogo e respeito, facilitando a corresponsabilização e satisfação do sujeito e de sua rede social ${ }^{4,19}$.

Em casa é melhor porque a gente podia mostrar, né, algum alimento que a gente tinha, qual era melhor, qual num era, qual que deveria usar (12).

As vezes que ele [marido] ficou, né, pra ele foi até bom porque ele tirou algumas dúvidas, perguntou algumas coisas, contou pra você algumas coisas que eu fazia (8).

A consistência das respostas foi confirmada quando se analisaram as variáveis quantitativas (Tabela 1). O grupo que recebeu as visitas domiciliares apresentou melhorias estatisticamente significativas na avaliação clínica, com redução dos parâmetros de IMC $\left(-0,7 \mathrm{~kg} / \mathrm{m}^{2} ; p=0,01\right)$; CC $(-4,2 \mathrm{~cm} ; p=0,001), P A$ sistólica $(-13 \mathrm{mmHG}$; $p=0,004)$ e glicemia $(-18,9 \mathrm{mg} / \mathrm{dl} ; p=0,01)$, enquanto o grupo que não recebeu orientações domiciliares teve redução apenas da CC $(-2 \mathrm{~cm}$; $p=0,01)$

Neste estudo, além da importância atribuída às atividades educativas pelas participantes, percebe-se também a valorização da presença de outros profissionais que atuam no PSF, além daqueles da equipe mínima, destacando-se o papel do nutricionista e as ações de educação nutricional.

A gente conseguiu entender você melhor, né, a sua explicação, você com aquele papel, aquele computador aquele dia, com tudo, tanta coisa mostrando. Você vê que com o médico, num é, né, eles falam só ali (9).

Estudos apontam a dificuldade de outros profissionais da saúde em realizar atividades de educação e orientação nutricional, uma vez que não têm formação adequada para essa função. 
Tabela 1. Variáveis quantitativas antes e após a intervenção nos grupos 1 (oficina) e 2 (oficina + orientação domiciliar). Porto Firme (MG), 2009.

\begin{tabular}{|c|c|c|c|c|c|c|}
\hline \multirow{3}{*}{ Variaveis } & \multicolumn{6}{|c|}{ Grupo 1 (oficina) } \\
\hline & \multicolumn{2}{|c|}{ Inicial (I) } & \multicolumn{2}{|c|}{ Final (F) } & \multirow{2}{*}{ Z } & \multirow{2}{*}{ pa } \\
\hline & M & $\mathrm{DP}$ & M & DP & & \\
\hline Peso, kg & 75,3 & 17,4 & 75,4 & 16,3 & $-0,126$ & 0,452 \\
\hline $\mathrm{IMC}, \mathrm{kg} / \mathrm{m}^{2}$ & 31,3 & 7,0 & 31,3 & 6,3 & $-0,157$ & 0,440 \\
\hline $\mathrm{CC}, \mathrm{cm}$ & 100,3 & 14,9 & 98,5 & 14,5 & $-2,100$ & $0,017^{*}$ \\
\hline PAS, $\mathrm{mmHg}$ & 119,3 & 14,9 & 117,4 & 15,9 & $-0,637$ & 0,261 \\
\hline $\mathrm{PAD}, \mathrm{mmHg}$ & 80,7 & 10,7 & 77,1 & 14,9 & $-0,962$ & 0,168 \\
\hline Glicose, mg/dL & 110,9 & 67,3 & 85,8 & 14,6 & $-1,083$ & 0,279 \\
\hline Triglicerídeos, mg/dL & 172,1 & 84,3 & 146,7 & 71,0 & $-0,464$ & 0,650 \\
\hline Colesterol total, mg/dL & 220,8 & 54,1 & 212,3 & 52,8 & $-0,035$ & 0,972 \\
\hline LDL-colesterol, mg/dL & 136,0 & 30,6 & 136,3 & 42,9 & $-0,175$ & 0,861 \\
\hline HDL-colesterol, mg/dL & 42,2 & 6,2 & 46,5 & 13,0 & 0,877 & 0,381 \\
\hline Per capita/dia óleo (ml) & 41,8 & 17,8 & 21,9 & 13,7 & $-2,500$ & $0,010^{*}$ \\
\hline Per capita/dia açúcar (g) & 106,9 & 67,5 & 85,8 & 70,1 & $-1,300$ & 0,170 \\
\hline \multirow[t]{2}{*}{ Per capita/dia sal (g) } & 11,0 & 8,4 & 8,6 & 5,6 & $-1,600$ & 0,100 \\
\hline & \multicolumn{6}{|c|}{ Grupo 2 (oficina + orientação domiciliar) } \\
\hline Peso, kg & 77,7 & 14,2 & 76,0 & 13,1 & $-2,098$ & $0,018^{*}$ \\
\hline $\mathrm{IMC}, \mathrm{kg} / \mathrm{m}^{2}$ & 33,4 & 5,7 & 32,7 & 5,3 & $-2,062$ & $0,019^{*}$ \\
\hline $\mathrm{CC}, \mathrm{cm}$ & 104,0 & 12,2 & 99,7 & 11,5 & $-2,986$ & $0,001^{*}$ \\
\hline $\mathrm{PAS}, \mathrm{mmHg}$ & 129,3 & 17,0 & 116,1 & 9,6 & $-2,631$ & $0,004^{*}$ \\
\hline $\mathrm{PAD}, \mathrm{mmHg}$ & 78,4 & 10,7 & 76,9 & 7,5 & $-0,486$ & 0,344 \\
\hline Glicose, mg/dL & 116,8 & 35,0 & 97,8 & 27,8 & $-2,552$ & $0,011^{*}$ \\
\hline Triglicerídeos, mg/dL & 147,3 & 72,8 & 131,3 & 51,5 & $-0,804$ & 0,421 \\
\hline Colesterol total, mg/dL & 203,5 & 56,2 & 193,3 & 30,1 & $-0,393$ & 0,695 \\
\hline LDL-colesterol, mg/dL & 123,5 & 48,6 & 117,4 & 24,8 & $-0,175$ & 0,861 \\
\hline HDL-colesterol, mg/dL & 50,9 & 8,8 & 49,5 & 9,3 & $-0,350$ & 0,727 \\
\hline Per capita/dia óleo (ml) & 35,9 & 16,0 & 21,4 & 10,9 & $-3,000$ & $0,002^{*}$ \\
\hline Per capita/dia açúcar (g) & 100,0 & 47,9 & 75,4 & 45,0 & $-2,200$ & $0,020^{*}$ \\
\hline Per capita/dia sal (g) & 10,6 & 6,4 & 8,1 & 2,6 & $-1,100$ & 0,270 \\
\hline
\end{tabular}

a $p$ valor para diferença entre valores antes e após intervenção para os grupos 1 (palestra) e 2 (visita) pelo teste de Wilcoxon. * $p<0,05 ;$ Os valores nas células se referem ao valor da Média (M), Desvio-Padrão (DP).

IMC: Índice de Massa Corporal; CC: Circunferência de Cintura; PAS: Pressão Arterial Sistólica: PAD: Pressão Arterial Diastólica; LDL: Lipoproteína de Baixa Densidade: HDL: Lipoproteína de Alta Densidade.

Além da falta de embasamento teórico para identificar e lidar com problemas nutricionais, evidencia-se a dificuldade desses profissionais em analisar os hábitos alimentares e seus aspectos subjetivos, uma vez que tratam da questão alimentar de forma inadequada 20,21 .

Ao salientarem a importância das atividades educativas e do nutricionista na prática dos serviços de saúde da família, as mulheres demonstraram preocupação com o coletivo ao destacarem a importância de as atividades educativas serem expandidas para o restante da população local e para outros municípios. Esse fato ficou mais evidente no grupo que recebeu as visitas domiciliares:

Esse trabalho aqui vai servir de exemplo pra outras cidades, né, que não têm essa oportunidade... que esse trabalho num pare aqui, pelo contrário, que ele continue crescendo, porque a quantidade de pessoas que é hipertensa aqui em Porto Firme é muito grande (8).

Diante da realidade encontrada, as diferentes percepções das mulheres sobre as atividades educativas compõem um espaço que relaciona a educação em saúde à promoção da saúde, 
que vai desde a perspectiva individual à coletiva, passando pelo vínculo profissional/usuário (Figura 1).

De fato, a disseminação da informação e a educação em saúde são fundamentais para a tomada de decisão e, por isso, componentes importantes da promoção da saúde, por estarem ligadas ao princípio de empoderamento e libertação de pessoas, famílias e coletividades ${ }^{18,22}$.

2. As mudanças nos hábitos alimentares: da (des)informação à conscientização - abrindo as portas à adesão.

A maioria (96\%) das entrevistadas afirmou ter realizado alguma modificação no hábito alimentar após o processo de capacitação nas duas modalidades de atividades educativas (Tabela 2). Houve diferença estatisticamente significante quanto à restrição à quantidade de alimento $(p=0,028)$ e diminuição do consumo de massas $(p=0,019)$ entre os dois grupos, destacando o

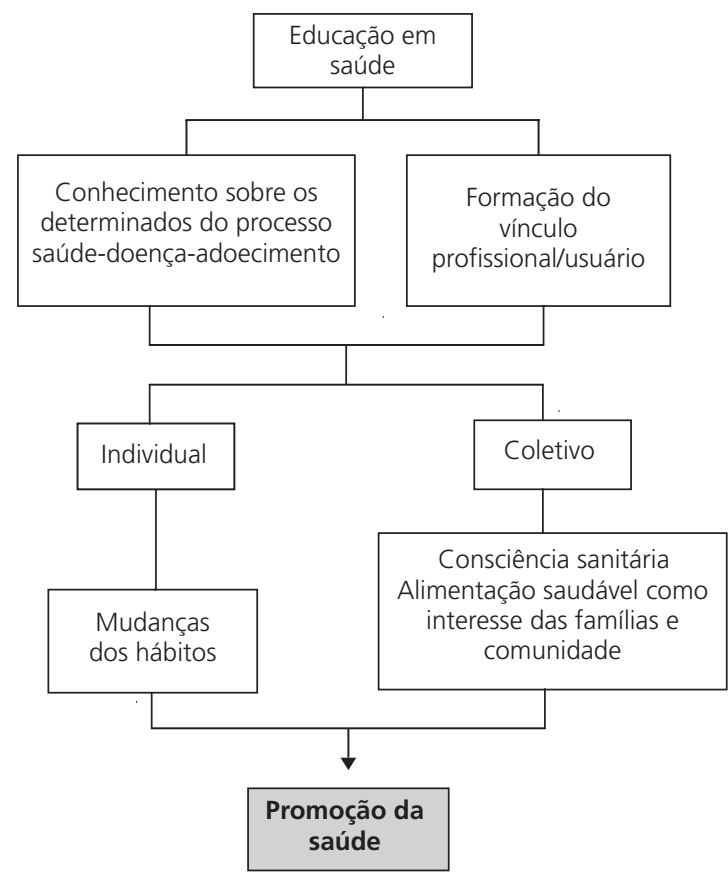

Figura 1. Relações construídas entre educação em saúde e promoção da saúde a partir da percepção de mulheres portadoras de hipertensão arterial sobre as atividades educativas. Porto Firme (MG), 2009. importante papel das atividades educativas, em especial das visitas domiciliares, para modificação dos hábiots alimentares.

Quanto à qualidade da dieta, em relação à percepção sobre as mudanças nos hábitos alimentares, os grupos tiveram mudanças semelhantes de comportamento: diminuição no consumo de sal e gordura/óleo e aumento do consumo de verduras, frutas e legumes, como as principais mudanças.

... diminuiu assim bastante a quantidade de óleo que eu usava... eu comprava nove litros de óleo, num dava pra quinze dias... hoje não, o óleo dá pra mais de mês (2).

As demonstrações de preparo e o porcionamento dos alimentos como técnicas que visavam ajudar as participantes a superarem as dificuldades no preparo das refeições foram apontados pelas mulheres dos dois grupos como importante fator para a compreensão e a consequente adesão às orientações sobre o uso de óleo/gordura; açúcar e sal.

[na oficina] Teve um dia que ela fez até uma demonstração lá com sal, o arroz e o óleo. Foi bom, a gente conscientizou, né (10).

[no domicílio] Mostrou a medida que nós usávamos e a medida certa que deve se usar, a quantidade no caso pra cada tipo de alimento que você ia usar ... a gente vai fazer um café, hoje eu sei a quantidade de açúcar (8).

O grupo que recebeu a orientação nutricional domiciliar aumentou significativamente o consumo de alimentos do grupo de alimentos protetores ou "neutros" $(p=0,01)$, especialmente em relação ao consumo de frango $(p=0,006)$ e cereais integrais $(p=0,002)$. No grupo das oficinas, houve aumento significativo no consumo de leite desnatado e semidesnatado $(p=0,03)$ e pão integral $(p=0,02)$, e diminuição do consumo de pele de porco/torresmo $(p=0,02)$ e suco artificial adoçado $(p=0,03)$. 
Tabela 2. Modificações nos hábitos alimentares de portadoras de hipertensão arterial sistêmica participantes de atividades educativas. Porto Firme (MG), 2009

\begin{tabular}{|c|c|c|c|c|c|c|}
\hline \multirow{2}{*}{ Modificações nos hábitos alimentares } & \multicolumn{2}{|c|}{ Grupo 1 (oficina) } & \multicolumn{2}{|c|}{ Grupo 2 (oficina + orientação domiciliar) } & \multirow{2}{*}{ Z } & \multirow{2}{*}{$P$} \\
\hline & $\mathrm{n}$ & $\%$ & $\mathrm{n}$ & $\%$ & & \\
\hline Restrição à quantidade de alimento & 2 & 15 & 8 & 57 & $-2,20$ & $0,028^{*}$ \\
\hline Diminuição do consumo de massas & 0 & 0 & 5 & 36 & $-2,34$ & $0,019^{*}$ \\
\hline Diminuição no consumo de sal & 10 & 77 & 13 & 93 & $-1,14$ & 0,253 \\
\hline Diminuição no consumo de gordura/óleo & 11 & 84 & 12 & 86 & $-0,79$ & 0,937 \\
\hline Aumento no consumo de verduras, frutas e hortaliças & 9 & 70 & 10 & 71 & $-0,12$ & 0,902 \\
\hline
\end{tabular}

a $p$ valor para diferença entre os grupos 1 (oficina) e 2 (oficina + orientação domiciliar) pelo teste de Mann Whitney. ${ }^{*} p<0,05$.

Quanto ao consumo de óleo, açúcar e sal (Tabela 1), no grupo das oficinas houve diferença estatisticamente significante apenas para o consumo per capita/dia de óleo $(p=0,01)$. Já no grupo das visitas domiciliares, houve diferença no consumo per capita/dia de óleo $(p=0,002)$ e açúcar $(p=0,02)$. Houve uma redução média de $14 \mathrm{~mL}$ no consumo per capita/dia de óleo e $27 \mathrm{~g}$ de açúcar. No grupo das visitas domiciliares, houve uma redução significativa no consumo per capita/dia dos indi-víduos que consumiam acima de $15 \mathrm{~g}$ de sal/dia ( $n=4)(p=0,03)$ : uma redução média de 9 , Desvio-Padrão $(D P)=1,3 g$ no consumo diário desse condimento.

Quanto à quantidade da dieta e à diminuição no consumo de massas, as mulheres que foram orientadas por visitas domiciliares alteraram significamente esses parâmetros. O acompanhamento individual permitiu trabalhar de forma mais detalhada questões como o fracionamento das refeições e as recomendações de cada grupo de alimentos, o que refletiu na diminuição do IMC e CC nesse grupo.

Em relação às variáveis clínicas e bioquímicas, a orientação domiciliar como estratégia de intervenção nutricional teve efeitos notadamente mais significativos. No presente estudo, não foi possível estabelecer estatisticamente a relação entre as mudanças dietéticas e as alterações nos parâmetros clínicos e bioquímicos avaliados nos distintos grupos de intervenção. Entretanto, o aumento do consumo de alimentos considerados protetores - ricos em fibras, vitaminas e minerais, e pobres em gordura e sódio - poderia explicar, pelo menos em parte, o resultado positivo sobre as variáveis clínicas, antropométricas e bioquímicas da intervenção no grupo que recebeu orientação domiciliar, conforme mostrado em estudos que relacionam padrões alimentares a fatores de risco para DCV 23,24 .

Ressalta-se que as mudanças referidas pelas mulheres foram associadas à melhorias em sua saúde, principalmente em relação ao sono, digestão e funcionamento intestinal; aumento da disposição e controle da PA.

Depois da alimentação, depois que mudei tudo... eu passei a dormir. Eu não dormia direito, acho que eu num tava alimentando do jeito que era pra alimentar (14).

... minha saúde melhorou muito. Minha pressão tá controlada, colesterol, a diabetes tá controlada (15).

No grupo que recebeu as visitas domiciliares, destaca-se a perda de peso, referida por $57 \%$ das mulheres como uma importante conquista, já que o excesso de peso oferece riscos à saúde.

A gente tem que procurar saber qual é $o$ limite de peso que a gente deveria estar... mesmo que a gente num fique muito dentro da tabela, mas pelo menos próximo, né. Eu tô tentando! (1).

Quanto aos obstáculos relacionados às mudanças nos hábitos alimentares, as mulheres mostraram dificuldades relativas à própria dieta, à questão financeira, à falta de disponibilidade 
de certos gêneros para a compra e à aceitação da família.

Quanto à qualidade da dieta, a mudança das escolhas alimentares - com a redução do consumo de alimentos ricos em açúcar, gordura/ óleo e sal - foi percebida de forma distinta. Enquanto algumas participantes atribuíam as mudanças à melhora no sabor dos alimentos, outras relatavam dificuldades relacionadas à quantidade de açúcar, por exemplo, e afirmavam seguir as recomendações por elas fazerem bem à saúde.

... igual eu gostava muito de um café bem docinho, aí foi difícil... leite também, esse leite desnatado não é gostoso, né, ai foi difícil também (5).

Outro fator limitante do seguimento das orientações pelas mulheres foi a questão financeira, sobretudo em relação à compra de determinados gêneros alimentícios, incluindo as frutas.

Mais dificuldade é quando a gente não tem o dinheiro pra comprar, porque nem todas frutas a gente pode comprar, né, todas as frutas é boa, mas é caro (13).

Dados da literatura mostram a influência de variáveis demográficas e socioeconômicas na determinação do consumo de frutas, verduras e legumes na população brasileira, sendo menor o consumo desses gêneros nas áreas rurais e nos estratos populacionais de menor escolaridade e renda ${ }^{25,26}$, como observado neste estudo.

As dificuldades relacionadas à família se referem ao fato de as mudanças na alimentação afetarem os outros membros do núcleo familiar, que nem sempre compreendem, aceitam ou aderem a essas mudanças.

O marido acaba sendo uma dificuldade porque... ele fala assim: o arroz podia ter um pouquinho mais de sal... homem é mais difícil (11).

Pelos depoimentos, no caso das mulheres que receberam orientações domiciliares e que envolveram os familiares no processo de aprendizagem, observa-se um comportamento mais positivo quanto às mudanças.
A orientação foi pra família, e o marido tá entrando também... agora a família toda pegou o ritmo meu lá. Graças a Deus! (2).

Considera-se que a motivação pessoal dos indivíduos em seguir as recomendações dietéticas pode ser prejudicada por falta de suporte da família ou amigos ${ }^{27}$.

\section{As atividades educativas na visão das participantes:}

A questão da adesão das portadoras de HAS envolveu a discussão de dois polos: "as percepções sobre as atividades educativas", que correspondem aos processos de mudança no nível cognitivo, representados pelo aumento da consciência sobre o processo saúde-doença-adoecimento e sobre a importância da alimentação na manutenção da saúde; e "as percepções sobre as mudanças alimentares", que correspondem ao processo de mudança comportamental e aos fatores que a influenciam.

Buscou-se dimensionar essas percepções nos diferentes subespaços que aproximam ou diferenciam as estratégias de educação em saúde propostas - as oficinas de educação em saúde e as visitas domiciliares -, de forma a analisar as peculiaridades dessas estratégias.

As representações gráficas desses subespaços estão demonstradas na Figura 2. Na parte superior da figura, que representa as percepções sobre as atividades educativas, verificam-se percepções comuns às oficinas e às visitas, uma vez que ambas pareceram apontar para o incremento da consciência das mulheres em como atuar em prol da própria saúde por meio da alimentação, contribuindo para o empoderamento. A importância do nutricionista como ator fundamental nesse processo também foi apontada pelas participantes.

Considerando que ambas as estratégias de educação em saúde buscaram romper com um modelo hegemônico de educação, acredita-se que 


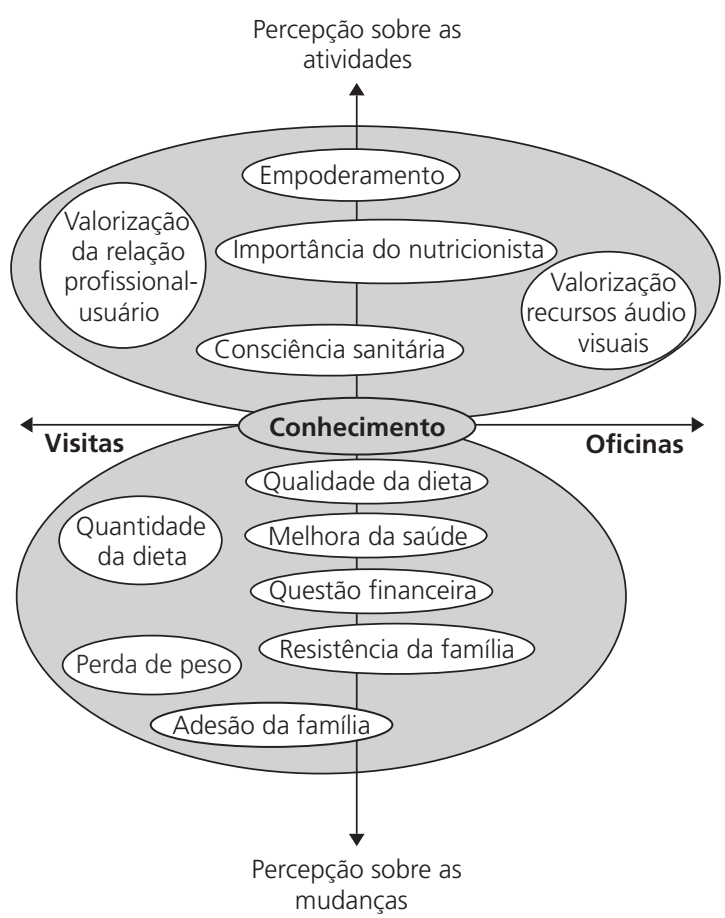

Figura 2. Subespaços construídos a partir da percepção das usuárias sobre as atividades educativas e as mudanças no comportamento alimentar. Porto Firme (MG), 2009.

as visitas domiciliares permitiram superar de forma mais concreta a fragmentação existente entre profissional de saúde e usuário, fortalecendo o vínculo.

Na parte inferior da figura, que representa as percepções das usuárias sobre as mudanças alimentares, ambos os grupos perceberam positivamente as mudanças na qualidade da dieta, associando essas mudanças à melhoria da saúde e à qualidade de vida. De toda forma, pode-se observar que as mulheres que receberam as visitas domiciliares expressaram a percepção de modificação quantitativa da dieta, associada à perda de peso, o que reforça a importância das orientações domiciliares por parte da equipe multiprofissional do PSF.

Como fator limitante para o seguimento das orientações, ressalta-se a questão financeira apontada pelas mulheres: esse aspecto deve ser avaliado pela equipe do PSF na elaboração de estratégias de intervenção e educação em saúde.
A família, como importante suporte social das usuárias, teve um papel ambivalente nas mudanças alimentares. Se, por um lado, para algumas mulheres a família foi associada à dificuldade na mudança dos hábitos alimentares, pela resistência às mudanças, por outro, ela também foi vista como parte ativa e integrante dessa mudança quando aderiu às orientações nutricionais, incentivando as portadoras de HAS. Esse paradoxo foi relatado em ambos os grupos, mas no grupo que recebeu as visitas domiciliares, observou-se uma maior participação da família no processo de mudança dos hábitos alimentares.

De fato, a visita domiciliar configura-se um espaço privilegiado para o contato e o desenvolvimento de atividades educativas com a família e outras pessoas significantes, que são o suporte social aos usuários e contribuem para a manutenção de hábitos saudáveis ${ }^{28}$.

Por fim, no centro da figura, o conhecimento destaca-se como elo entre as atividades educativas e as mudanças de comportamento nas duas estratégias, uma vez que é visto pelas usuárias como elemento potencialmente capacitador de mudanças. Ainda que o conhecimento por si só não signifique mudança, ele constitui o primeiro degrau do comportamento saudável, sendo instrumento para mudança22.

\section{O N C L US Ã O}

As estratégias educativas constituem um importante instrumento de acesso ao conhecimento sobre o processo saúde-doença-adoecimento, aumentando a capacidade de controle sobre os determinantes desse processo. O vínculo entre pesquisador/profissional e usuário mostrou-se um elo fundamental na integração entre as políticas públicas de saúde e a comunidade.

As orientações domiciliares especializadas tiveram efeito positivo significativo sobre o processo educativo das portadoras de HAS, evidenciado pela percepção das participantes e pelas mudanças nas variáveis clínicas, bioquímicas, 
antropométricas e de consumo alimentar antes e após a intervenção. Reforça-se a importância das orientações domiciliares por permitirem, além da aproximação do profissional à realidade familiar (ambiente físico, material e afetivo) do portador de HAS, a possibilidade de se vivenciarem concretamente a rotina de preparação das refeições - quantidade e qualidade dos alimentos - e as relações dessas mulheres e dos familiares com os alimentos.

Destarte, destacam-se a necessidade do trabalho multiprofissional e interdisciplinar para lidar com o portador de HAS e o cuidado domiciliar (home care) como uma estratégia privilegiada para maior adesão ao tratamento da doença.

\section{COLABORADORES}

AG RIBEIRO e RMM COTTA participaram da concepção do idealização do trabalho, da pesquisa bibliográfica, da metodologia, da coleta, da análise, da discussão e da redação do artigo. LS SILVA, SMR RIBEIRO, CMGC DIAS, SM MITRE e MCF NOGUEIRA-MARTINS participaram da coleta, da análise, da discussão e da redação do artigo.

\section{REFERÊ NCIAS}

1. Brasil. Ministério da Saúde. Hipertensão arterial sistêmica. Brasília: MS; 2006.

2. Fuchs SC, Castro MS, Fuchs FC. Adesão ao tratamento anti-hipertensivo: análise das evidências. Rev Bras Hipertens. 2004; 7(3):90-3.

3. Reiners AAO, Azevedo RCS, Vieira MA, Arruda ALF. Produção bibliográfica sobre adesão/não-adesão de pessoas ao tratamento de saúde. Ciên Saúde Colet. 2008; 13(Supl 2):2299-306. doi: 10.1590/S 1413-81232008000900034.

4. Sá LD, Souza KMJ, Nunes MG, Palha PF, Nogueira JA, Villa TCS. Tratamento da tuberculose em unidade de saúde da família: história de abandono. Texto Contexto Enferm. 2007; 16(4):712-8. doi: 10.1590/S0104-07072007000400016.

5. Aubert L, Bovet P, Gervasovi JP, Rwebogora A, Waeber B, Paccaud F. Knowledge, attitudes, and practices on hypertension in a country in epidemiological transition. Hypertension. 1998; 31:1136-45. doi: 10.1161/01.HYP.31.5.1136.
6. Costa GD, Cotta RMM, Ferreira MSLM, Monteiro CA. Saúde da família: desafios no processo de reorientação do modelo assistencial. Rev Bras Enferm. 2009; 62(1):113-8. doi: 10.1590/S0034-7 1672009000100017.

7. Cotta RMM, Batista KCS, Reis RS, Souza GA, Dias GD, Castro FAF, et al. Perfil sociossanitário e estilo de vida de hipertensos e/ou diabéticos, usuários do Programa de Saúde da Família - Município de Teixeiras - MG. Ciên Saúde Colet. 2009; 14(4): 1251-60. doi: 10.1590/S1413-8123200900040 0031.

8. Ribeiro AG, Ribeiro SMR, Dias CMGC, Ribeiro AQ, Castro FAF, Suárez-Varela MM, et al. Nonpharmacological treatment of hypertension in primary health care: a comparative clinical trial of two education strategies in health and nutrition. BMC Public Health. 2011; 11:637. doi: 10.1186/14 71-2458-11-637.

9. Minayo MCS, Assis SG, Souza ER. Avaliação por triangulação de métodos. Rio de Janeiro: Fiocruz; 2005.

10. Minayo MCS. O desafio do conhecimento: pesquisa qualitativa em saúde. $10^{a}$ ed. São Paulo: Hucitec; 2007.

11. Bardin L. Análise de conteúdo. Lisboa: Edições 70; 2004.

12. Ribeiro AC, Sávio KEO, Rodrigues MLCF, Costa THM, Schmitz BAS. Validação de um questionário de freqüência de consumo alimentar para população adulta. Rev Nutr. 2006; 19(5):553-62. doi: 10.1590/S1415-52732006000500003.

13. Jellife DBI. Evaluación del estado de nutrición de la comunidad. Genebra: OMS; 1968.

14. World Health Organization. Physical status: the use and interpretation of anthropometry - report of a WHO Expert Commitee. Geneva: WHO; 1995. WHO Technical Report Series 854.

15. World Health Organization. Obesity: preventing and managing the global epidemic. Geneva: WHO; 1998. Report of a WHO Consulation on Obesity.

16. Sociedade Brasileira de Cardiologia. V Diretrizes Brasileiras de Hipertensão Arterial. Arq Bras Cardiol. 2007; 89(3):24-79.

17. Fornés NS, Martins IS, Velásquez-Meléndez G, Latorre MRDO. Escores de consumo alimentar e níveis lipêmicos em população de São Paulo, Brasil. Rev Saúde Pública. 2002; 36(1):12-8. doi: 10.1590/S 0034-89102002000100003.

18. Cotta RMM, Reis RS, Carvalho AL, Siqueira KC, Castro FAF, Alfenas RCG. Reflexões sobre o conhecimento dos usuários no contexto do Programa de Saúde da Família: a lacuna entre o saber técnico e o popular. Physis. 2008; 18(4):745-66. doi: 10.15 90/S0103-73312008000400008. 
19. Pinheiro R, Mattos RA, organizadores. Construção da integralidade: cotidiano, saberes e práticas em saúde. Rio de Janeiro: Abrasco; 2003.

20. Boog MCF. Atuação do nutricionista em saúde pública na promoção da alimentação saudável. Ciên Saúde Colet. 2008; 1(1):33-42.

21. Santos AC. A inserção do nutricionista na estratégia da saúde da família: o olhar de diferentes trabalhadores da saúde. Fam Saúde Desenv. 2005; 7(3): 257-65.

22. Brasil. Ministério da Saúde. Secretaria de Políticas de Saúde. As cartas da promoção da saúde. Brasília: MS; 2002.

23. Champagne CM. Dietary interventions on blood pressure: the dietary approaches to stop hypertension (DASH) trials. Nutr Rev. 2006; 64(2):S45-S56.

24. Vollmer WM, Sacks FM, Ard J, Appel LJ, Bray GA, Simons-Morton DG, et al. Effects of diet and sodium intake on blood pressure: subgroup analysis of the DASH-sodium trial. Ann Intern Med. 2001; 135:1019-28.
25. Claro RM, Carmo HCE, Machado FMS, Monteiro CA. Renda, preço dos alimentos e participação de frutas e hortaliças na dieta. Rev Saúde Pública. 2007; 41(4):557-564. doi: 10.1590/S0034-89102 007000400009.

26. Jaime PC, Monteiro CA. Consumo de frutas e hortaliças na população adulta brasileira, 2003. Cad Saúde Pública. 2009; 21(Supl.1):19S-24S. doi: 10.1590/S0102-311X2005000700003.

27. Horn LVV, Dolecek TA, Grandits GA, Skweres L. Adherence to dietary recommendations in the special intervention group in the Multiple Risk Factor Intervention Trial. Am J Clin Nutr. 1997; 65(suppl. 1):289S-304S.

28. Araújo GB, Garcia TR. Adesão ao tratamento antihipertensivo: uma análise conceitual. Rev Eletrônica Enferm [Internet]. 2006 [acesso 2010 nov 28]; 8(2): 259-72. Disponível em: <http://www.fen.ufg.br/ revista/revista8_2/v8n2a11.htm>.

Recebido em: 9/5/2011

Versão final em: 9/3/2012

Aprovado em: 23/3/2012 